\title{
Effect of Beam Diameter on the Accurate Analysis of Silicate Glasses using Electron Probe Microanalysis
}

\author{
Ruo-Xi Zhang ${ }^{1}$, Shui-Yuan Yang ${ }^{1 *}$, Shao-Yong Jiang ${ }^{1,2}$, Jing Xie ${ }^{3}$ \\ 1. State Key Laboratory of Geological Processes and Mineral Resources, China University of \\ Geosciences, Wuhan, PR China. \\ 2. Faculty of Earth Resources and Collaborative Innovation Center for Scarce and Strategic Mineral \\ Resources, China University of Geosciences, Wuhan, PR China. \\ 3. Key Laboratory of Continental Collision and Plateau Uplift, Institute of Tibetan Plateau Research, \\ Chinese Academy of Sciences, Beijing, PR China. \\ * Corresponding author: shuiyuanyang@cug.edu.cn
}

Electron probe microanalysis (EPMA) is a widely used analytical technique which is mainly used for quantifying the contents of the major, minor, even trace elements of solid materials. EPMA is also well known as its non-destructive characteristic. However, electron beam irradiation will cause permanent damage to beam-sensitive materials, such as glass, as well as the migration of mobile components (e.g., alkalis and volatile), leading to time-dependent intensity (TDI) changes of elemental characteristic Xrays [1]. In particular, sodium migration under the electron bombardment can be significant, which is enabled by local heating and electron energy by the incident electron beam [2] or electric field-assisted diffusion [3]. When unsuitable beam condition is used, it will lead to underestimate the content of $\mathrm{Na}$, and overestimate that of relative immobile elements, for example, Si and Al.

These issues mentioned above are typically overcome by use of low beam currents and large beam diameters (e.g., $2 \mathrm{nA}$ and $20 \mu \mathrm{m}$ ) [4-6]. However, intensities of characteristic X-rays may be lost with the large beam diameter. The characteristic X-ray intensities of elements in silicate glasses were determined using a JEOL JXA-8100 electron probe microanalyzer at the State Key Laboratory of Geological Processes and Mineral Resources, China University of Geosciences (Wuhan). Beam current of $20 \mathrm{nA}$ with a TAP crystal and a PET crystal, and variant beam diameters were used on olivine and jadeite at $15 \mathrm{kV}$ to find out the effects of beam diameters on characteristic X-ray intensity (Fig. 1). Along with the increasing beam diameters, when $>30 \mu \mathrm{m}$, the detected characteristic X-ray intensities of elements start to drop off (Fig. 1a). Besides, as can be seen from Fig.1a, even though $\mathrm{Na}, \mathrm{Mg}, \mathrm{Al}, \mathrm{Si}$ were analyzed under the same condition, $\mathrm{Si}$ suffered a more serious decline in characteristic X-ray intensity along with the increasing beam diameter whereas that of $\mathrm{Na}$ was less severe than others. These may be caused by X-ray spectrometer defocusing (out off of the Rowland Circle) for some of the outer perimeter of the large beam.

Most critically, incorrect results with low analytical totals and underestimation of the content of elements arise if a large beam diameter is conducted on specimens but the standards are conducted at a routine beam condition (much smaller beam diameter). The experiment result shows a consistency of degrees of characteristic X-ray intensity loss of an identical element at the same analytical condition on different matrixes (Fig. 1b). Therefore, we recommend that when elements are analyzed using a TAP or PET crystal and using a beam diameter larger than 30, standards and specimens should be analyzed with the same beam diameter, which is not only for the analysis of glasses but also all the solid materials in EPMA. Further research needs to be conducted to find out the effect of beam diameter of other crystals. 
Overall, the reasonable selection of beam condition is necessary for EPMA of silicate glasses. It would be better if a liquid nitrogen cold trap is attained, which also helps to remove heat from the analyzed area [7-8]. Besides, the element diffusion rate will be reduced significantly by moving the specimen during analysis [9]. Although moving the specimen around during analysis involves a large homogenous area, it does reduce, even eliminate, the effect of characteristic X-ray intensity drop-off by Na migration. Because of the time-dependent nature of $\mathrm{Na}$ migration, it should be determined at the beginning of an analysis session to minimize the effects of its mobilization. Hence, it is necessary to balance count-times and accumulation of adequate counts [7]. When a lower beam current is used, it will lower the characteristic X-ray intensity generated and data points become scattered, leading to uncertainty and deviation of the results. Therefore, using a low beam current is not a feasible approach for elements with low concentrations. A higher beam current provides more precise and reliable results, but it calls for a large beam diameter. Our study shows that beam condition of $5 \mathrm{nA}$ and $20 \mu \mathrm{m}$ works well for the analysis on NIST SRM-610, NIST SRM-612, NMNH 72854 and NMNH 2231 glasses, with $\mathrm{Na}_{2} \mathrm{O}$ range from $1.06 \mathrm{wt} \%$ to $13.7 \mathrm{wt} \%$. When the grain is big enough, it is advantageous to use a relatively high beam current with a larger beam diameter as it helps to reduce Na migration and improve statistical accuracies through high counts. However, the same beam diameter must be used for both the standard and the specimen to optimize the data acquisition [10].

\section{References:}

[1] GB Morgan and D London, Am. Miner. 8 (1996), p. 1176.

[2] LF Vassamillet and VE Caldwell, J. Appl. Phys. 10 (1969), p. 1643.

[3] DM Usher, J. Phys. C: Solid State Phys. 14 (1981), p. 204.

[4] CH Nielsen and H Sigurdsson, Am. Miner. 66 (1987), p. 548.

[5] GB Morgan and D London, Am. Miner. 90 (2005), p. 1131.

[6] SC Kuehn, DG Froese and RAR Shane, Quat. Int. 246 (2011), p. 20.

[7] JG Spray and DA Rae, Can. Miner. 33 (1995), p. 330.

[8] B Buse and S Kearns, Microsc. microanal. 21 (2015), p. 604.

[9] Y Ono et al., Microbeam Anal. 20 ${ }^{\text {th }}$ Annu. Conf. (1985) p. 421.

[10] The authors acknowledge funding from the National Key R\&D Plan of China (No.

2017YFC0601404) and the National Natural Science Foundation of China (No. 41773040).
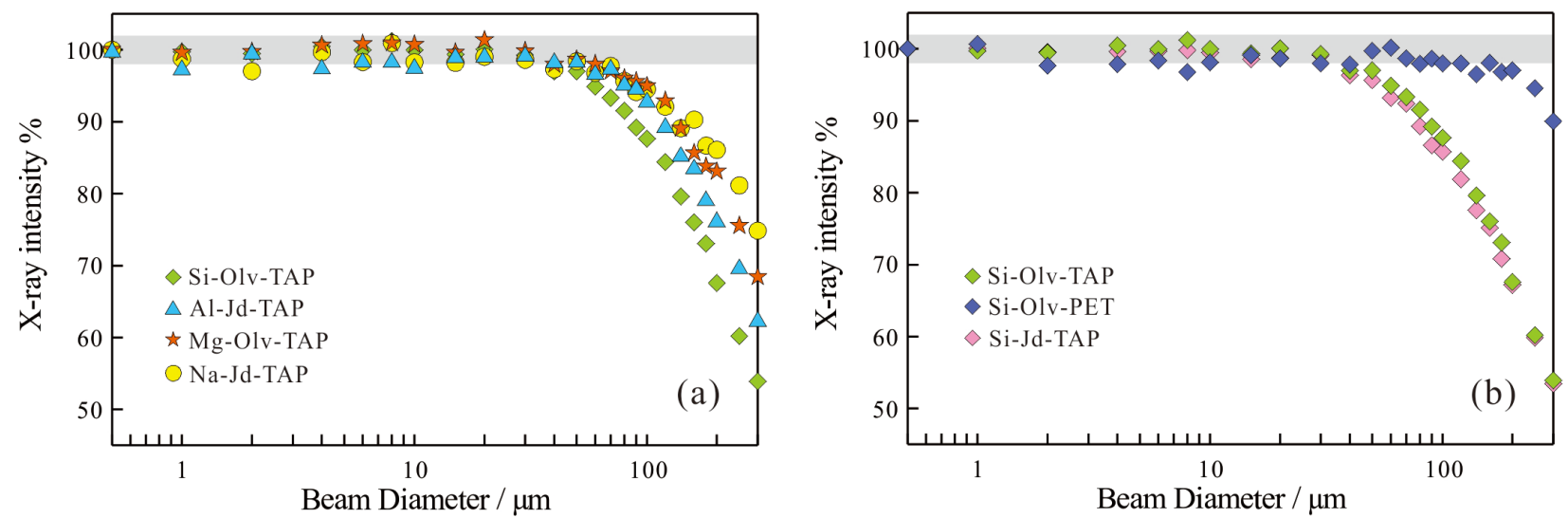

Figure 1. Graph showing with the increasing beam diameter, the detected characteristic X-ray intensity changes of (a) $\mathrm{Na}, \mathrm{Mg}$, Al and Si with a TAP crystal and (b) Si in olivine and jadeite with a TAP and a PET crystal. The gray area in the graph ranges from $98 \%$ to $102 \%$ on the vertical axis. 